T cells in flow-based adhesion assays. Total adhesion of CD4 and CD8 T cells was significantly $(p<0.05)$ reduced when ICAM- 1 , VCAM-1, and CXCR3 molecules were blocked or if G protein coupled receptors were inhibited with pertussis toxin (PTX). CD8 T cell adhesion was also dependent on vascular adhesion protein-1.

Conclusion We report high IL-18 expression by Kupffer cells in inflammatory liver disease. The ability of IL-18 to enhance T cell recruitment via sinusoidal endothelium suggests it acts to promote lymphocyte recruitment during the development of chronic hepatitis and is thus a potential novel therapeutic target in inflammatory liver disease.

Competing interests None declared.

\section{PMO-121 OSTEOPONTIN PROMOTES NATURAL KILLER T (NKT) CELL ACCUMULATION IN NONALCOHOLIC STEATOHEPATITIS (NASH)}

doi:10.1136/gutjnl-2012-302514b.121

${ }^{1} \mathrm{E}$ Liaskou, ${ }^{* 1} \mathrm{~L} \mathrm{C}$ Claridge, ${ }^{1,2} \mathrm{R}$ Younis, ${ }^{1} \mathrm{H}$ Shah, ${ }^{1} \mathrm{~J}$ Shaw, ${ }^{1} \mathrm{Y} \mathrm{H}$ Oo, ${ }^{3} \mathrm{Z} \mathrm{Mi},{ }^{3} \mathrm{P} \mathrm{C}$ Kuo, ${ }^{4} \mathrm{~A}$ Canbay, ${ }^{1} \mathrm{D}$ H Adams, ${ }^{5,6} \mathrm{~A}$ M Diehl, ${ }^{1,2} \mathrm{~W}$ K Syn. ${ }^{1}$ Centre for Liver Research, University of Birmingham, Birmingham, UK; ${ }^{2}$ Regeneration and Repair, Institute of Hepatology, London, UK; ${ }^{3}$ Department of Surgery, Loyola University, Chicago, USA; ${ }^{4}$ Department of Gastroenterology, Essen University, Essen, Germany; ${ }^{5}$ Department of Medicine, Duke University, Durham, USA; ${ }^{6}$ Department of Gastroenterology, Duke University, Durham, USA

Introduction Progressive steatohepatitis is characterised by increased inflammatory cell infiltration. Osteopontin (OPN) is a cytokine intricately associated with immune-cell accumulation, and we reported that NKT cells accumulate to promote hepatic injury in NASH. We hypothesise that OPN promotes NASH progression by supporting NKT migration across hepatic sinusoidal endothelium. Methods Mice were fed chow or methionine-choline deficient (MCD) diet to induce NASH. After 4 weeks, mice were sacrificed; severity of disease assessed by serum aminotransferase (AST), liver OPN quantified by ORTPCR and immunohistochemistry, blood OPN measured by ELISA. In separate studies, MCD-fed mice were treated with sham or OPN aptamers, and FACS used to quantify liver NKTs. Primary human hepatic sinusoidal endothelial cells (HSEC) were stimulated with recombinant (r)OPN (0-1000 ng/ $\mathrm{ml})$, with or without TNFa $(20 \mathrm{ng} / \mathrm{ml})+\operatorname{IFNg}(100 \mathrm{ng} / \mathrm{ml})$, and expression of adhesion molecules (ICAM1, VCAM1) and chemokines (CXCL9, 10, 11, 16) assessed. To assess lymphocyte migration, lymphocytes were perfused over rOPN-or vehicle-treated-HSEC, with or without TNFa+IFNg. In separate experiments, TNFa + IFNg stimulated-HSEC were treated with OPN aptamers or blocking antibodies, and total lymphocyte adhesion recorded. Human NASH livers were immunostained for OPN, plasma measured for OPN, and liver NKT numbers from normal or NASHcirrhotic patients quantified by FACS.

Results In mice, MCD-induced NASH upregulated expression of liver OPN by threefolds $(p<0.05)$, blood OPN by twofolds $(p<0.05)$, increased liver CD4 by 2.2-fold, and NKT cells by threefold $(p<0.05)$. $\mathrm{MCD}$-fed mice treated with OPN aptamers accumulated fewer CD4 and NKT cells $(p<0.05)$, and exhibited attenuated injury (ALT: twofold reduction; $\mathrm{p}<0.02)$. rOPN induced expression of ICAM-1, VCAM-1 and CD31 on human HSEC, enhanced lymphocyte recruitment under conditions of flow (40\%), and amplified recruitment ability of TNFa+IFNg stimulated HSEC (20\%), while OPN neutralisation with RNA-aptamers reduced lymphocyte recruitment by $50 \%($ all $p<0.05)$. OPN effects were mediated via CD $44, a_{v} b_{3}$, ICAM1, VCAM1, and CXCL16. In humans, liver and plasma OPN was significantly upregulated in NASH; livers from NASH-cirrhosis harboured twofolds more CD4 and threefolds more NKT cells $(p<0.05)$ than normal.

Conclusion Liver and plasma OPN levels are upregulated during steatohepatitis in mice and humans, and promote liver NKT accumulation. OPN neutralisation significantly reduces lymphocyte subset recruitment and liver injury, suggesting that OPN is a promising anti-inflammatory target in steatohepatitis.

Competing interests None declared.

\section{PM0-122 EICOSAPENTAENOIC ACID IS EFFECTIVE AT REDUCING HEPATOCYTE TRIGLYCERIDE CONTENT OF UNTREATED C3A CELLS BUT IS NOT EFFECTIVE IN TWO MODELS OF CELLULAR STEATOSIS}

doi:10.1136/gutjnl-2012-302514b.122

G Masterton, * P Cowan, P Lee, E Pryde, P C Hayes, J N Plevris. Division of Clinical and Surgical Sciences, University of Edinburgh, Edinburgh, UK

Introduction Eicosapentaenoic acid (EPA), one of the major physiologically active constituents of Omega- 3 fatty acids, has been suggested as a treatment for non-alcoholic fatty liver disease (NAFLD). The aim of these experiments was to assess the effects of EPA on intrahepatic triglyceride content in cell culture models of steatosis.

Methods Human C3a hepatocytes were incubated in MEME (standard media) and two models of cellular steatosis: oleate (a model of isolated steatosis) and LPON (a model of steatosis and mitochondrial dysfunction containing the gluconeogenic substrates Lacate, Pyruvate, Octanoate and ammoNia). Test media was either unsupplemented, or supplemented with $50 \mu \mathrm{M}$ or $250 \mu \mathrm{M}$ EPA. Hepatocyte triglyceride accumulation was assessed both by microscopy (using oil red staining) and by quantifying the intracellular triglyceride concentration of cells incubated in culture media for 3 and 7 days. Each cell culture experiment was performed in triplicate.

Results $\boldsymbol{M E M E}$ When quantified by oil red staining a $73.1 \%(95 \% \mathrm{CI}$ $63 \%$ to $83 \%$ ) reduction in cell triglyceride content with $250 \mu \mathrm{M} \mathrm{EPA}$ compared with untreated cells was seen (7659 vs 28564 pixels; $\mathrm{p}<0.001)$. This was confirmed in cell culture experiments as $250 \mu \mathrm{M}$ EPA was associated with reduced intrahepatocyte triglyceride content after both 3 (74.1 vs $94.9 \mathrm{mmol} / \mathrm{gTP} ; \mathrm{p}<0.05)$ and 7 days (62.2 vs $80.9 \mathrm{mmol} / \mathrm{gTP} ; \mathrm{p}<0.05)$ incubation compared with untreated cells equating to a $21.9 \%$ (95\% CI $9 \%$ to $35 \%$ ) and $23.1 \%$ (95\% CI $5 \%$ to $41 \%)$ reduction respectively. For both experiments a linear trend between increasing EPA concentration and reduced triglyceride content was confirmed. Oleate Here reduced triglyceride content with both $50 \mu \mathrm{M}$ EPA $(\mathrm{p}<0.01)$ and $250 \mu \mathrm{M}$ EPA $(\mathrm{p}<0.05)$ was seen with oil red staining and equated to reductions of $27.6 \%$ (95\% CI $16 \%$ to $39 \%$ ) and $22.5 \%$ (95\% CI $9 \%$ to $36 \%$ ) compared with untreated cells. However these results were not reproduced in cell culture experiments although on post test analysis there was a significant linear trend between increasing EPA concentration and reduced triglyceride content $(p=0.04)$. LPON Although incubation with $250 \mu \mathrm{M}$ EPA reduced triglyceride content in the LPON model when quantified with oil red staining (60308 vs 79219 pixels in $250 \mu \mathrm{M} \mathrm{EPA}$ vs untreated cells; $p<0.05)$ this was not confirmed in cell culture experiments. On post hoc analysis no trend was demonstrated between EPA concentration and triglyceride content.

Conclusion These results suggest that EPA is effective at reducing triglyceride accumulation in untreated hepatocytes but is not effective in either oleate or LPON models of cellular steatosis. It is therefore possible that the presence of steatosis and mitochondrial dysfunction in NAFLD may limit the efficacy of EPA as a treatment.

Competing interests None declared. 